\title{
A Concept for Monolithic Shape Memory Alloy Micro-Devices
}

\author{
Y. Bellouard, R. Clavel, J.-E. Bidaux*, R. Gotthardt** and T. Sidler*** \\ Institut de Microtechnique, DMT, École Polytechnique Fédérale de Lausanne, 1015 Lausanne, \\ Switzerland \\ * Laboratoire de Technologie des Composites et Polymères, DMX, École Polytechnique Fédérale \\ de Lausanne, 1015 Lausanne, Switzerland \\ ** Institut de Génie Atomique, DP, École Polytechnique Fédérale de Lausanne, 1015 Lausanne, \\ Switzerland \\ *** Institut d'Optique Appliquée, DMT, École Polytechnique Fédérale de Lausanne, 1015 Lausanne, \\ Switzerland
}

\begin{abstract}
Most of Shape Memory Alloy (SMA) devices use only the SMA material as the actuating part or in some cases as the flexible part of the device (superelastic hinges). Commonly, simple wires, strips or springs are used for the actuating part. All these systems need an assembly procedure which raises several problems in the micro-system field : careful assembly, sometimes friction, .... The concept of monolithic SMA micro-devices presented in this paper is to integrate all functions of the device within the same material. For example, in a micro-gripper, the jaws and the moving part are made out of the same piece of material. With this technology, we avoid any assembly in the small dimensions. Moreover sub-millimeter devices can easily be designed and further miniaturization can be achieved. As an illustration, two micro-devices based on this concept are presented.
\end{abstract}

\section{INTRODUCTION}

Most SMA micro-devices described in the literature are made up of several mechanical parts (bearings, bias springs, jaws, frame, ....) and the SMA actuator is only used as the actuating part of the system. For instance, Ikuta uses two antagonist SMA springs for actuating each arm of his gripper [1]. In another way, Hesselbach and al. [2] propose a gripper design based on flexible hinges actuated by an SMA fiber. The "bias spring" used to achieve a reversible effect is the elasticity of the structure itself. Generally speaking, all these designs need an assembly which is hardly realizable in very small dimensions. Our main purpose was to develop monolithic SMA devices in which the moving part, the fixation part and the actuator are all included into the same piece of material. Based on this technique, we had developed two kinds of microdevices. The first one - a fastening system - uses the One-Way-Shape-Memory-Effect and the second one a micro-gripper - uses the Two-Way-Shape-Memory-Effect. Miniaturization has been obtained in both cases as the dimension of each device does not exceed one half of a millimeter.

\section{THE APPLICATION}

This work took place in an industrial project in which the goal was to design new technologies in automated assembly of flexible micro-endoscopes which are used in mini-invasive surgery. These microendoscopes are mainly made up of a multicore fiber and Selfoc $\circledast$ micro-lenses.

One of the major tasks was to develop a gripper dedicated to the grasping and handling of micro-lenses. However, handling this kind of objects raises various problems :

- The lens shape is cylindrical and the size does not exceed half a millimeter.

- The lenses are brittle and must be handled with extreme care.

- As the lens has to be used for fine positioning, the optical axis must remain available. 
- To ensure good image quality, care must be taken to avoid contamination by dust or water

- The dimensional tolerances of the lens are wide (20\%).

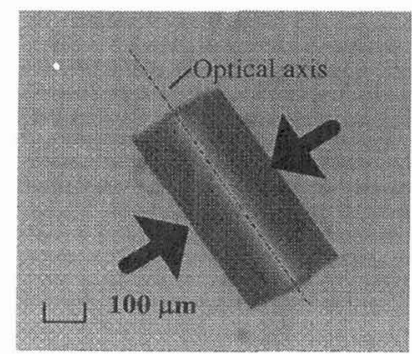

Fig 1 grasping area on a micro-lens. (dimensions are $:$ height $=\mathbf{5 0 0} \mu \mathrm{m}$, Radius $=\mathbf{2 5 0} \mu \mathrm{m}$ )

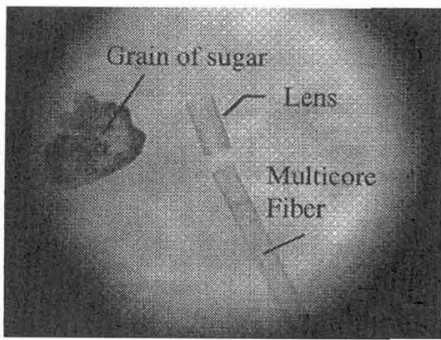

Fig 2 Components of an endoscope (a grain of sugar has been added to give an idea of dimensions)

Moreover, during the assembly process, we also needed a lens holder and a fastening system, for clipping the illuminating fibers around the micro-endoscope (fig. 3):

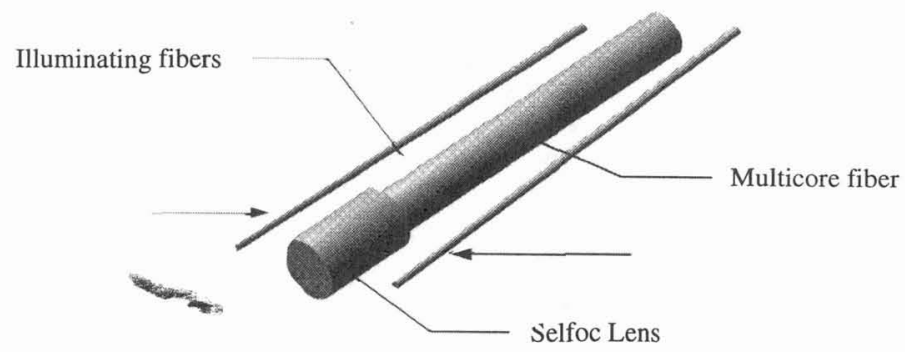

Fig. 3 - Schematic representations of a flexible micro-endoscope

On the basis of these specifications, the shape memory material was the best solution.

\section{THE MICRO-FASTENER}

This micro-fastening device design is based on a ring-like shape whose radius changes with temperature (see Fig 4).
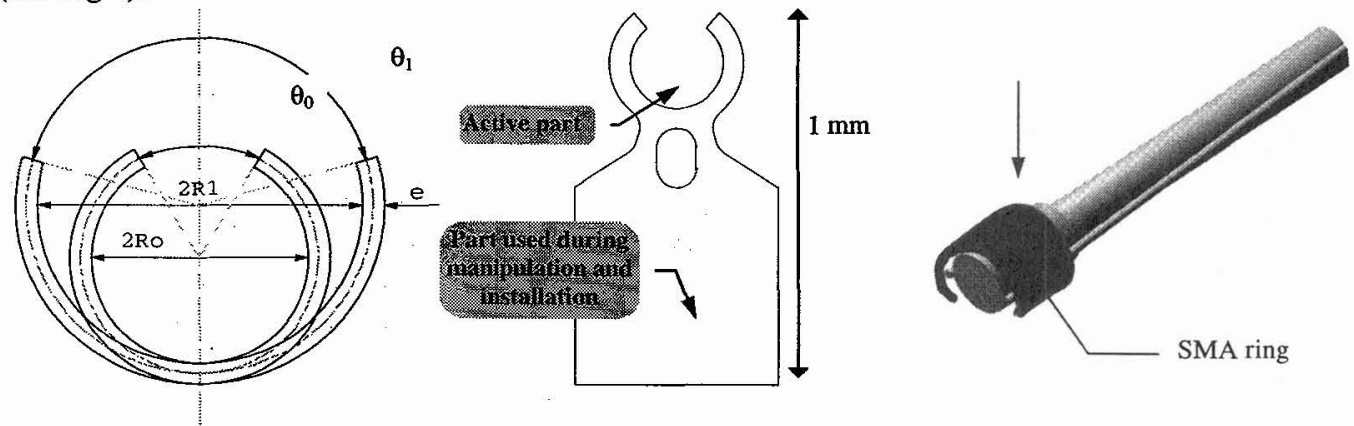

Fig 4 The SMA micro-fastener : principle, real design and application. 
This design should be described by three parameters (fig 4$)$ : the thickness (e), the initial radius $\left(\mathrm{R}_{0}\right)$ and the opening angle $\left(\theta_{0}\right)$.

\section{Calculus}

Hypothesis :

- We consider an ideal ring neglecting the effect of fixation of the gripper itself.

- The strain is supposed to be equal, at the most, to $4 \%$ in One Way Shape Memory Effect.

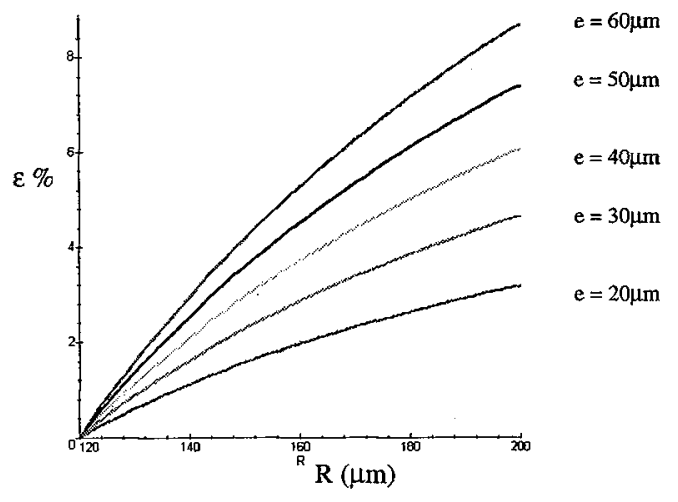

Fig 5 Maximum strain vs radius for five different thickness. (Initial radius $\mathbf{R}_{\mathbf{0}}=\mathbf{1 2 0} \boldsymbol{\mu m}$ )

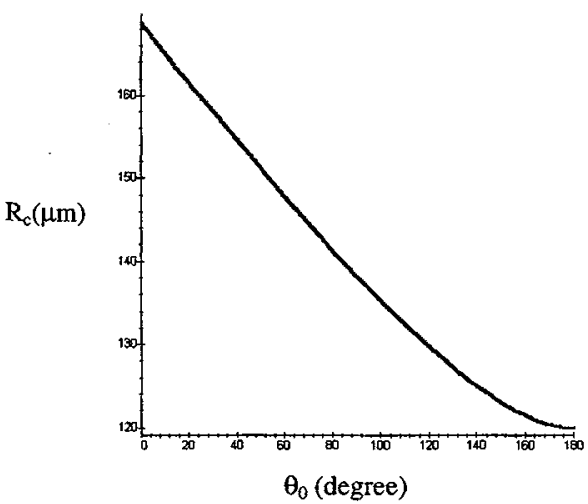

Fig 6 Critical radius (Rc) vs opening angle $\left(\theta_{0}\right)$ $\mathbf{R}_{0}=\mathbf{1 2 0} \mu \mathrm{m}$ and $\mathrm{e}=\mathbf{5 0} \mu \mathrm{m}$

Given $R_{1}$ the open radius, the maximum strain is written [3] :

$$
\varepsilon=\frac{e}{2}\left(\frac{1}{R_{0}}-\frac{1}{R_{1}}\right)\left(\frac{1}{1+\frac{e}{2 R_{\mathrm{I}}}}\right)
$$

To be able to insert an object from the top of the gripper, the minimum opening radius must be greater than a critical radius $(\mathrm{Rc})$ which is defined as the minimum radius to insert an object from the top of the ring. By defining $R_{o b j}$ the radius of the object which will be grasped, the following equation can easily be demonstrated [4]:

$$
\frac{R_{o b j}}{R_{c}}=\sin \left[\left(\pi-\frac{\theta_{0}}{2}\right)\left(\frac{R_{o b j}+\frac{e}{2}}{R_{c}+\frac{e}{2}}\right)\right]
$$

The solution of this equation is graphically represented on fig. 6 .

By choosing an initial radius and a thickness, we can calculate the strain and estimate the angle of opening needed for a vertical insertion. 


\section{THE MICRO-GRIPPER}

The micro-gripper (Fig.7) is based on the same principle described above. The shape is a "crab-like tweezers" one and to achieve a good accuracy, the grasped object is referenced on a V groove shape. The reversible motion is obtained by a training process which leads to the Two-Way-Shape-Memory-Effect. Heating and cooling are done by Peltier element.
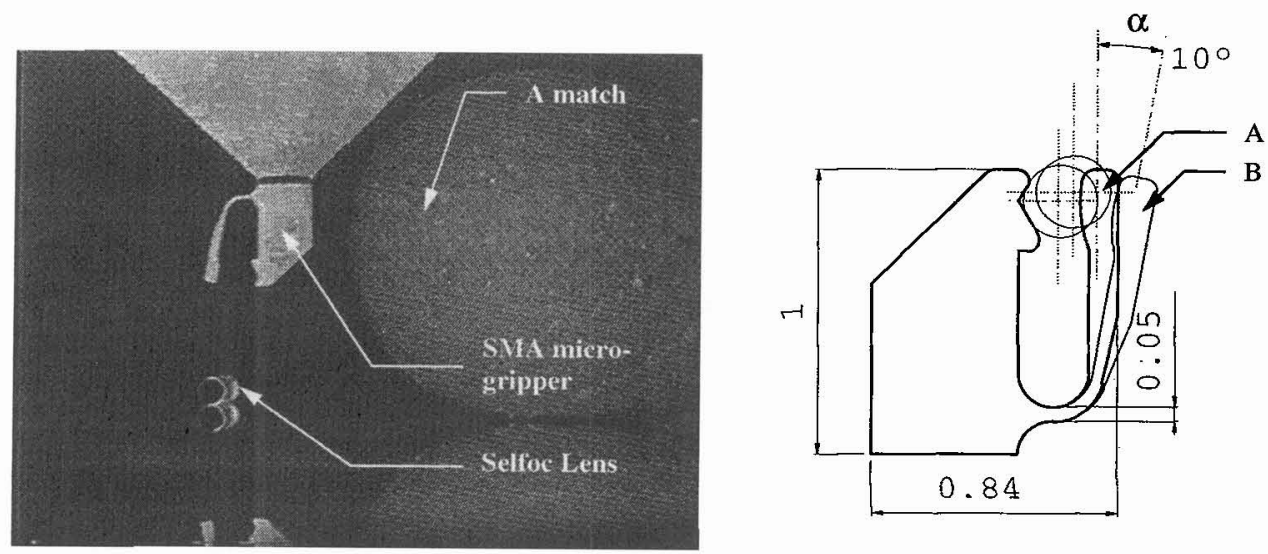

Fig 7 a) the micro-gripper

b) the design : A - Austenitic shape, B - Martensitic shape.

\section{Designing}

Let $R_{0}$ be the martensitic radius and $R_{1}$ the austenitic radius of the hinge.

Let $\mathrm{e}$ be the thickness of the hinge and $\alpha$ the angular position of the arm.

By assuming a pure bending deformation of the hinge, the martensitic radius writes :

$$
R_{1}=\frac{R_{0}}{1-\frac{2 \alpha}{\pi}}
$$

Thus the maximum strain can be written (1):

$$
\varepsilon=\frac{\alpha e}{\pi R_{0}}\left(\frac{1}{1+\frac{e}{2}\left(\frac{\pi-2 \alpha}{\pi R_{0}}\right)}\right)
$$

In the simulation (Fig.7 b), a strain of $2 \%$ for the two way shape memory effect (TWSME) was assumed. 


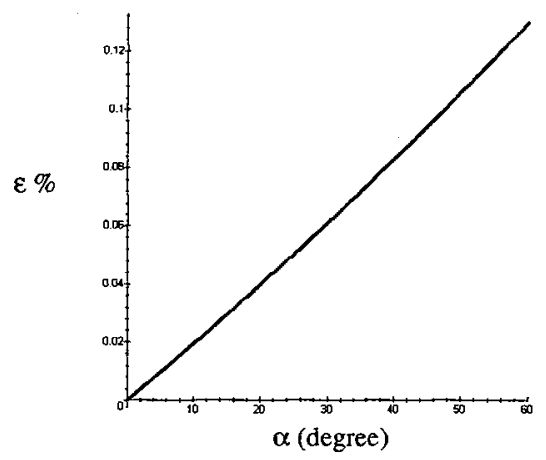

Fig 8 Strain versus angular position of the gripper arm

Three training methods were tested $[4,5]$ :

- Training by pseudo-elastic cycling

- Training by shape memory cycling

- Training by constrained temperature cycling of deformed martensite.

These three methods were tested for 5 and 10 cycles in each case. The simplest method is the constrained temperature cycling of deformed martensite. First results by using this method give us an angle between 6.3 to 7.5 degree according to Eq. 4 corresponds to 1.2 to $1.4 \%$ reversible strain.

\section{MATERIAL AND FABRICATION}

\subsection{The material}

The SMA material used in this application was a $200 \mu \mathrm{m}$ thick NiTiCu strip. The material was annealed for 10 min at $480^{\circ} \mathrm{C}\left(\mathrm{Ms}=48^{\circ} \mathrm{C}, \mathrm{Mf}=32^{\circ} \mathrm{C}\right.$, As $=63^{\circ} \mathrm{C}$, Af $\left.=82^{\circ} \mathrm{C}\right)$

\subsection{The laser cutting}

The material was laser-cut by a Nd Yag Slab Laser working in a fundamental mode (TEM 00). To increase the precision of cutting, the target was moved by a PC controlled 2 axes $\mathrm{CNC}$, under a fixed focusing objective. Dispersion in size was thus of the order of $2-3 \mu \mathrm{m}$. The main parameter for quality of cutting is the additional gas. In fact oxygen is the only gas, which avoids the re-adherence of the cut groove. Depending on the pressure of the gas, corrections of the outline have to be taken into account.

\section{CONCLUSIONS}

A new concept of SMA gripper and fastening system has been presented. These devices have been successfully used as a lens holder and as a gripper for " pick and place » tasks on micro-lenses and will be integrated in a production chain this year. Experiments show us that we can obtain a valuable two-way shape memory effect to pick and release objects inside the gripper. 


\section{Acknowledgments}

We would like to thanks Luca Mumenthaler for the cutting of the gripper, Dr. Ramiro Conde, Dr Ch. Depeursinge for their useful and wise advice in optical measurements and finally, Michel Sartirani for the testing under « real conditions ».

\section{References}

[1] K. Ikuta, "Microminiature Shape Memory Alloys Actuator", IEEE Int. Conf. On Robotics and Automation, Cincinatti, 1990, pp. 2156-2161.

[2] J. Hesselbach, R. Pittschellis, E. Hornbogen, M. Mertmann, "Shape Memory Alloys for use in miniature grippers", $2^{\text {nd }}$ international conference on Shape Memory and Superelastic Technologies, Monterey, 1997 (to be published).

[3] M. Thier, A. Mick, D. Drescher, C. Bourauel, "Deformation behaviour of NiTi shape memory alloys in bending”, Journal of Materials Science 26 (1991) pp 6473-6478.

[4] Y. Bellouard, R. Clavel, J.-E. Bidaux, R. Gotthardt, T. Sidler, "A new design of Shape Memory Alloy micro-gripper used in micro-optics assembly", $2^{\text {nd }}$ international conference on Shape Memory and Superelastic Technologies, Monterey, 1997 (to be published)

[5] J. Perkins, D. Hodgson, "The Two Way Shape Memory Effect", Engineering Aspect of Shape Memory Alloys, ed. by T.W. Duerig, et al, Butterworth-Heinmann, 1990, pp. 195-206. 